\title{
Congenital Heart Block in Lupus Erythematosus Syndrome and Anti Ro-SSA Antibodies. A Case Report
}

\section{Maria Caterina Corigliano*, Pizzicaroli Caterina and Larciprete Giovanni}

Fatebenefratelli Isola Tiberina Hospital, Italy

*Corresponding author: Maria Caterina Corigliano, Fatebenefratelli Isola Tiberina Hospital, Via di Ponte Quattro Capi 39, 00186, Rome, Italy.

\section{Introduction}

Congenital heart block is often associated with maternal anti Ro/SS-A and anti La/SSB antibodies that cross the placenta and damage the fetal AV node [1]. It occurs during the second trimester of gestation in asymptomatic women [2].

\section{Case Report}

In 2018 a 30-year-old, second gravida, was referred to the Department of Obstetrics, Ospedale San Giovanni Calibita,
Fatebenefratelli, tertiary care Centre of Rome, for fetal AV block at 35 weeks of gestation.

Autoimmune screening was performed and it revealed positivity to anti La/SS-A, anti-ANA, anti-RNP, anti-SM. She was not assuming corticosteroids.

Previous ultrasounds revealed at the first trimester screening an increased Nuchal Translucency and in the second trimester a IIdegree AV block.

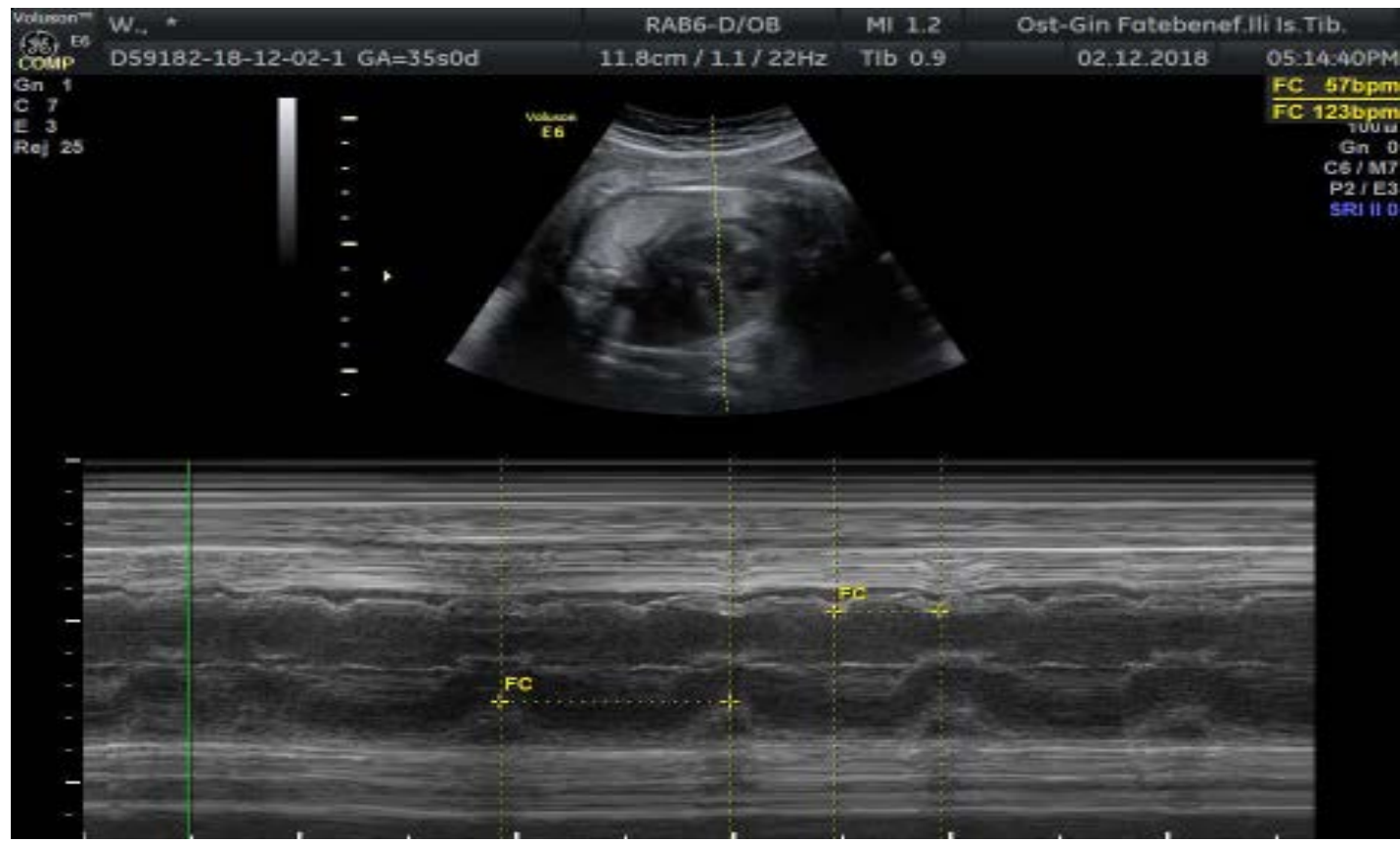

Video 1: M-mode with the $\mathrm{M}$ line passing through the right atrium and the left ventricle. It shows an atrial rate of $57 \mathrm{bpm}$ and a ventricular rate of $123 \mathrm{bpm}$. 


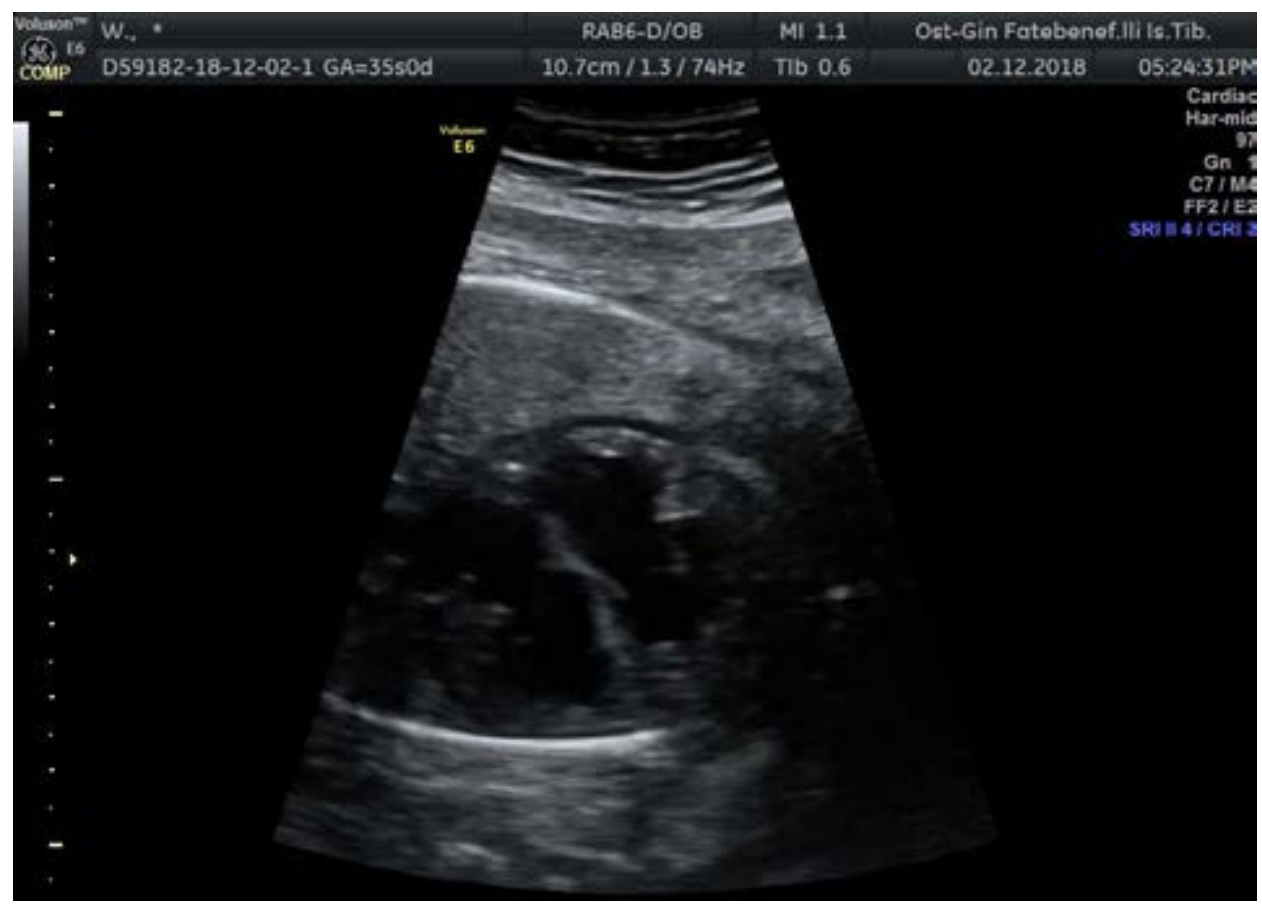

Video 2: B-Mode shows a complete dissociation between atrial and ventricular contractions.

The obstetric ultrasound and echocardiography detected a contractions (regular atrial rate of $130 \mathrm{bpm}$ and a persistent structurally normal heart with a third-degree complete AV block ventricular rate of $55 \mathrm{bpm}$ ) (Figure 1, Video 1 and Video 2). with a complete dissociation between atrial and ventricular

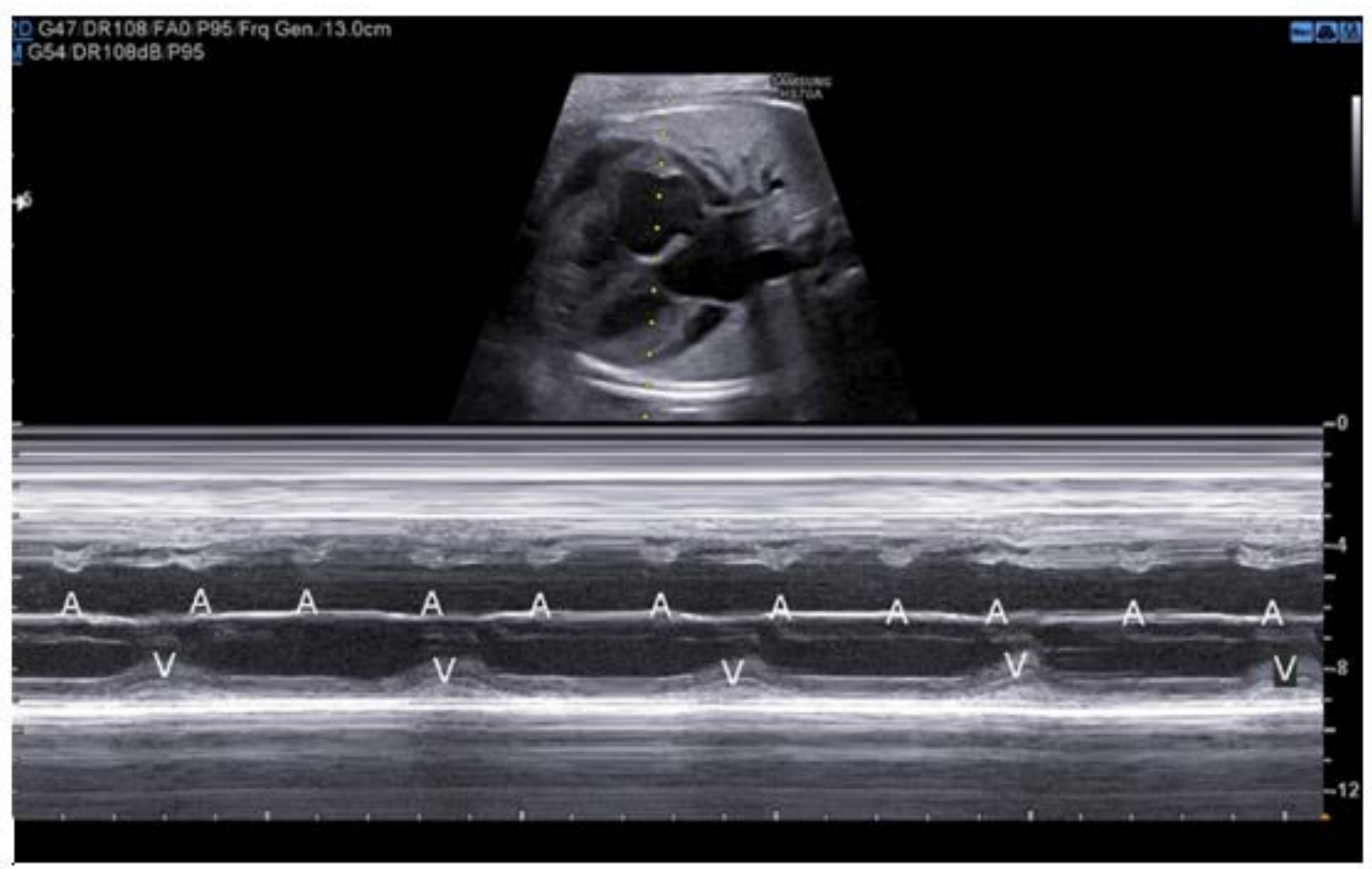

Figure 1: M-mode tracing of both the right atrium and the left ventricle. A: atrial contractions; $\mathrm{V}$ : ventricular contractions " $\mathrm{V}$ " at a much slower rate with evident atrio-ventricular dissociation.

Other findings included: cardiomegaly heart/thoracic area (CTA) ratio 0,4 , reduced heart function, mitral and tricuspid regurgitation, $7 \mathrm{~mm}$ pericardial effusion, ductus venosus reverse flow, pulsatile umbilical vein, normal diastolic flow in umbilical and MCA artery (PIUA: 1,1 PIMCA:1,91), EFW: 2750 gr (50 pc) AFI: 115. 

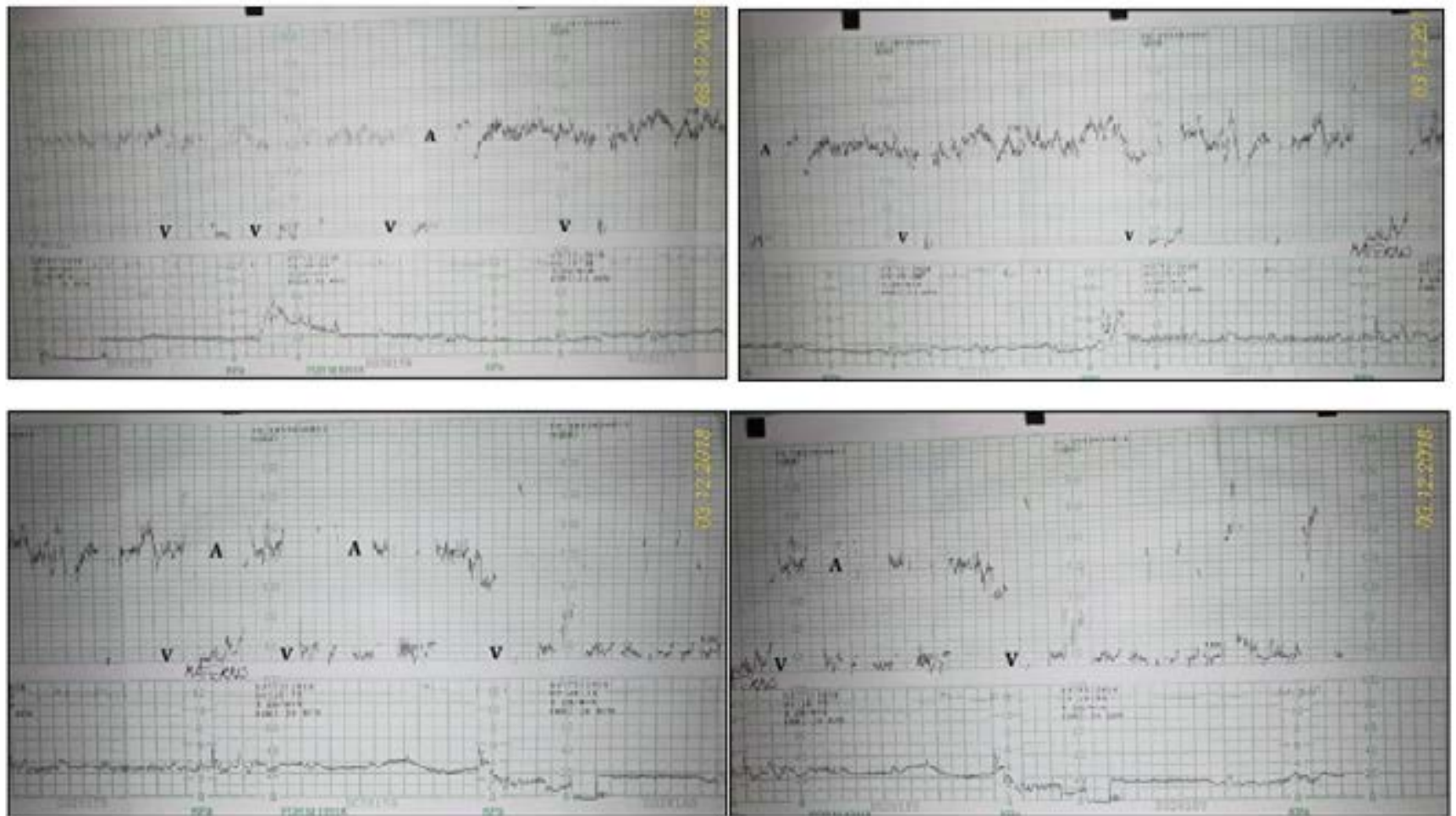

Figure 2: Cardiotocography monitoring shows an atrial base line of $130 \mathrm{bpm}$ and a ventricular base line of $60 \mathrm{bpm}$.

An occasional cardiotography revealed the complete AV block (Figure 2).

A subsequent caesarean section was performed. A 2800 gr baby was born. The complete AV block was confirmed at birth and a cardiac pacing was performed.

\section{Conclusions}

Lupus erythematosus syndrome affects pregnancy with maternal and fetal/neonatal complications that could be screened and prevented by a close monitoring of pregnancy.

Autoimmune screening with serum anti Ro/SS-A and anti La/ SSB antibodies should be performed to rule out the risk of fetal heart block.

In patients with anti Ro/SS-A and anti La/SSB antibodies regular prenatal ultrasounds after the second trimester of pregnancy should be planned to detect:

1. Congenital heart block. Maternal steroids may be used in first- and second-degree heart block to prevent the progression in a complete heart block. Anyway, systematic prophylactic therapy is not recommended [2].
2. Early fetal abnormalities (cardiac anatomy and function, fetal biophysical profile, fetal growth, fetal doppler assessment, hydrops) in order to plan the delivery in a tertiary care center where pacemaker placement could be performed.

\section{Acknowledgement}

We would like to thank so much our colleagues Valerio Romano and Gerardo Piacentini for their warm, deep technical support and for their echo-cardio-expertise.

\section{Conflict of Interest}

No conflict of interest.

\section{References}

1. Bloomenthal D, Wilson RD (2000) Systemic lupus erythematosus in pregnancy. JOGC 22(12): 1042-1047.

2. Rein AJ, Mevorach D, Perles Z, Gavri S, Nadjari M, et al. (2009) Early diagnosis and treatment of atrioventricular block in the fetus exposed to maternal anti-SSA/Ro-SSB/La antibodies: a prospective, observational, fetal kinetocardiogram-based study. Circulation. 119(14): 1867-1872. 